\title{
Development and application of streakline visualization in hypervelocity flows
}

\author{
P. Lemieux, H.G. Hornung
}

\begin{abstract}
A method for visualizing streaklines in hypervelocity flows has been developed. The method uses the high temperatures produced in hypervelocity flows to ablate small amounts of sodium deposited onto a wire stretched across the flow and to broaden the lines in the sodium spectrum. By using a dye laser, tuned to a wavelength close to one of the sodium D-lines, as the light source in shadowgraph or Schlieren visualization, streaklines seeded with sodium become visible through absorption and/or enhanced refractivity. The technique has been used to investigate the stability of the shear layer produced by the curved bow shock on a cylindrically blunted wedge. The results suggest that the shear layer is unstable, exhibiting structures with a wavelength that is comparable to half the nose radius of the body.
\end{abstract}

\section{1}

\section{Introduction}

A curved shock produces a shear layer with smoothly varying vorticity. The vorticity generated at the shock is a function of the shock angle, curvature, and density ratio across the shock (Hayes and Probstein 1966). A study was undertaken to investigate the stability of such shear layers, as produced by the curved bow shock on a blunt wedge in hypervelocity flows. One of the goals of this study was to use flow visualization techniques capable of highlighting instabilities in the shear layer, if these were found to exist. The idea was to duplicate the success of the smoke-wire in similar studies in subsonic flows.

In the traditional smoke-wire setup, the streak lines consist of smoke generated by oil that vaporizes as it runs down a heated wire placed upstream of a model in a wind tunnel. Provided that the wind tunnel flow has sufficiently low turbulence, this method can be used even at supersonic speeds (Goddard et al. 1959). In stability studies in particular, the smoke-wire has proved to be a valuable research tool and is widely used in qualitative and quantitative studies (e.g., Fric and Roshko 1994). A review of the literature, however, does not reveal any successful implementation of a similar technique in the short-duration flows of hypervelocity shock tunnels.

\section{2}

\section{Streakline visualization in hypervelocity flows}

Visualization using smoke is not realizable at the flow conditions typical of shock tunnels. On the other hand, Germain (1994) has been successful in applying the atomic sodium seeding method of Blendstrup et al. (1979) to visualize boundary layer flows at these conditions and provided the basis for a new flow visualization technique.

The new technique, called sodium wire streakline visualization (SWSV), combines the method of Blendstrup et al. (1979) with the smoke-wire idea. It uses the hot gas flow of the shock tunnel to produce streaks by ablation of sodium from a wire. By tuning a narrow bandwidth light source to a frequency close to an absorption line, streaks containing sodium may be visualized through a combination of absorption and enhanced refractivity, depending on the difference between the line center and light source frequencies.

The seeding is made relatively safe and simple by the high temperature of hypervelocity flow, as it enables sodium to be introduced in the form of sodium chloride, deposited at discrete source points on a steel wire stretched across the flow. The hot gas ablates and dissociates the sodium chloride, thus seeding the test gas continuously with atomic sodium emanating from the wire. Typical conditions encountered in the stagnation region of the wire are $7,000 \mathrm{~K}$ and $100 \mathrm{kPa}$, at which, in equilibrium, sodium chloride is $99 \%$ dissociated. Thus, the technique works because of either or both of the following effects:

- The absorption at or near one of the strong atomic sodium lines ( $D_{1}$ at $589.593 \mathrm{~nm}$ and $D_{2}$ at $588.996 \mathrm{~nm}$ ).

- The enhanced refractivity of sodium gas near such a line, which can be several orders of magnitude higher than that of air.

\section{Light absorption}

As has been shown by Blendstrup et al. (1979), enhanced refractivity and absorption are remarkably strong effects in sodium and concentrations of one part in 1,000 or even 10,000 are sufficient to produce the effects necessary for visualization. The effect of the seeding on the flow is therefore negligible. At the conditions of hypervelocity flow, the Doppler broadening of the line (resulting from the thermal motion of the atoms) is very strong, so that the 
tuning accuracy required is much reduced. Other linebroadening mechanisms are unimportant at our conditions (pressure, electric or magnetic broadening), so that the line shape may be taken to be Gaussian, as if produced by Doppler effects alone.

\section{3}

\section{Experimental setup}

The shock tunnel facility where SWSV was developed and used is the T5 Hypervelocity Shock Tunnel at the Graduate Aeronautical Laboratories of the California Institute of Technology. T5 is a free-piston shock tunnel, in which the driver gas is heated adiabatically by piston compression. The transient heating achieved by this method permits operation at higher driver gas temperatures. Descriptions of the operation of piston-driven shock tunnels in general are available in the literature (Lukasiewicz 1973; detailed descriptions of the T5 facility specifically may be found in Bélanger 1993, Germain 1994, and Wen 1994).

Three shock tunnel operating conditions were selected for the experiments performed with SWSV. These are listed in Table 1, along with their corresponding test section freestream conditions and expected equilibrium normal shock density ratios, and span a large range of reservoir enthalpies and free-stream conditions available in the facility.

\section{1}

\section{Experimental test of the absorption technique}

In a preliminary series of experiments aimed at substantiating the concept of SWSV, a wire, seeded with sodium chloride, was stretched in front of a sphere. This setup was placed in the T5 test section and subjected to flow condition 1 in Table 1 . An example of the results of this study, shown in Fig. 1, illustrates that the method clearly works, but also that the flow is easily contaminated by excess sodium on the wire.

Further tests were performed using a 4-in.-diameter cylinder at the same flow conditions as in Fig. 1. The wire was seeded at intervals of approximately $25 \mathrm{~mm}$; each site consisted of a salt crystal of roughly $3 \mathrm{~mm}$ in length and a fraction of a millimeter in thickness around the wire. The resulting flow is shown in Fig. 2.

While results such as this showed that streaklines can be traced using this technique and would provide a valuable tool in the study of the stability of the shear layer produced by the shock, further experiments showed that placing the wire downstream of the shock significantly improved the repeatability and reliability of the technique and made the seeding of specific areas of the flow easier.

\section{2}

\section{Experimental test of the enhanced refractivity method}

Streaklines may also be visualized by using the enhanced refractivity near a spectral line. Positive and negative refractivity peaks occur at approximately half a half-width on either side of the absorption peak of a spectral line. Tuning the light source to the refractivity peak thus can make techniques that rely on refractive-index gradients (Schlieren, differential interferometry) much more sensitive, so that they will easily pick up streaklines even though absorption is much reduced at this wavelength.
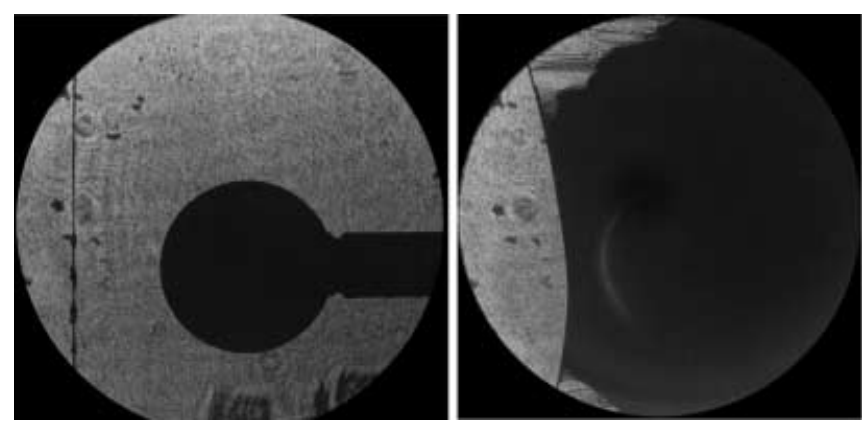

Fig. 1. Test of the SWSV concept. Flow is from left to right. Left: Model before the shot, a 3 inch diameter sphere with a wire seeded with sodium chloride at various locations. Right: Effect of the hot gas flow on the salt, creating a sheet seeded with atomic sodium which absorbs all the light downstream of the wire. The luminosity of the flow in the stagnation region of the sphere is just visible. The drag on the wire causes it to bend in the direction of the flow. The marks in the field of view upstream of the wire and below the sphere in the left figure are due to spots on the optical windows and are not part of the flow

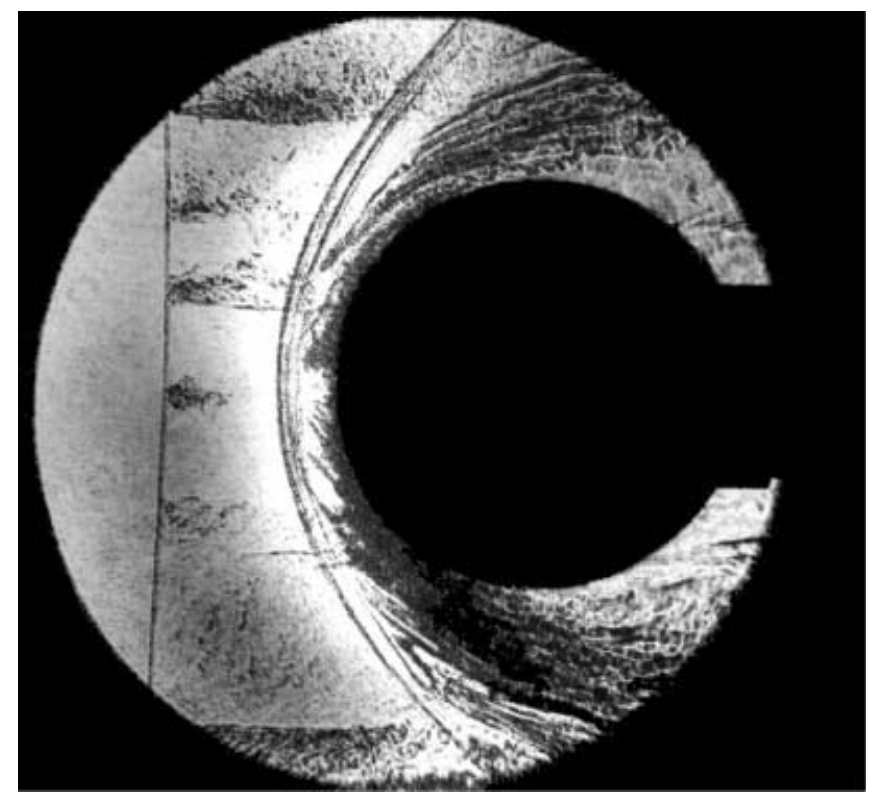

Fig. 2. Streaklines through the shock produced by a cylinder. The laser is tuned sufficiently far from the center of the line, so that there is no excessive light absorption. Note that the higher temperature of the flow downstream of the shock causes the sodium to absorb more light in that region than upstream of the shock
Table 1. Nominal T5 conditions and approximate density ratios for SWSV study

\begin{tabular}{lllllllr}
\hline & $h_{\text {res }}(\mathrm{MJ} / \mathrm{kg})$ & $P_{\text {res }}(\mathrm{MPa})$ & $M_{\infty}$ & $U_{\infty}(\mathrm{m} / \mathrm{s})$ & $\rho_{\infty}\left(\mathrm{kg} / \mathrm{m}^{3}\right)$ & $T_{\infty}(\mathrm{K})$ & $\rho_{2} / \rho_{1}$ \\
\hline Condition 1 & 11 & 20 & 5.7 & 4,300 & 0.018 & 1450 & 8.5 \\
Condition 2 & 20 & 27 & 5.0 & 5,500 & 0.018 & 3020 & 10.8 \\
Condition 3 & 4 & 14 & 6.2 & 2,700 & 0.036 & 580 & 6.2 \\
\hline
\end{tabular}




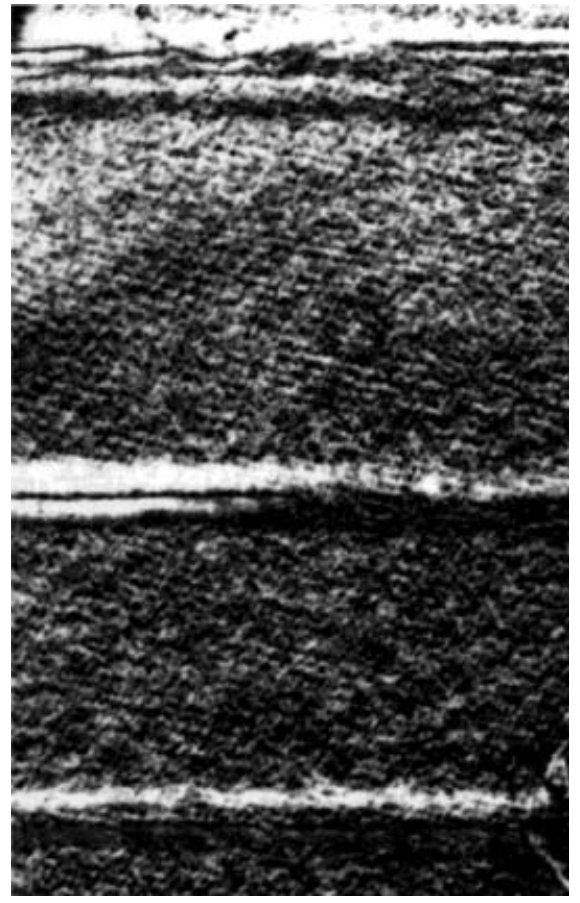

Fig. 3. Three streaks, visualized by enhanced refractivity of sodium with differential interferometry

An example is shown in the visualization of three freestream streaklines at test condition 1 (Fig. 3). The streaks are made visible by Wollaston prism differential interferometry, without noticeable absorption. The streaks in Fig. 3 would not have appeared in absorption shadowgraphy at the wavelength of the light source used. The enhanced refractivity method is less sensitive to the light source wavelength than absorption shadowgraphy.

\section{3}

\section{Apparatus, optical setup, and the development} of the flow-tagging technique

The light source for this experiment consists of a $300-\mathrm{mJ} /$ pulse, frequency-doubled Nd:YAG laser, which is used to pump a dye laser made by Cummings (1995), with the wavelength adjustable in the 587-594-nm range. These lasers were used as light sources for both shadowgraphy and differential interferometry.

The light source frequency was tuned to the edge of one of the sodium absorption lines, as it appeared in a propane flame seeded with sodium, at a temperature of approximately $1,600 \mathrm{~K}$ and atmospheric pressure. Using a formulation by Thorne (1988), the Doppler half-width of the sodium D-line may be calculated. At this condition, it was found to have a half-width of approximately $3.5 \mathrm{pm}$, whereas, at an assumed flow temperature of 7,000 K immediately downstream of the normal shock on the wire, the half-width is more than doubled to $7.4 \mathrm{pm}$.

Thus, though minimal absorption appears to be taking place through the propane flame, the absorption level would be perceptible, but not excessive, in typical flow conditions which would be somewhere between these two temperatures. The variation of both the refractive index and the absorption coefficient with wavelength in the line is fairly large and depends on the broadening of the line. Fine wavelength adjustments of the dye laser thus become necessary to obtain a given level of absorption and to avoid excessive absorption such as that shown in Fig. 1.

Figure 4 illustrates the method used to tune the laser. The laser light illuminates a screen after passing through a propane flame seeded with sodium. The level of absorption taking place is evident by observing the shadow of the flame on the back wall: with the laser frequency adjusted outside of the absorption line, the light passes through the flame with little or no absorption, and no shadow (except for the apparatus) appears on the wall; as the frequency of the laser is adjusted to within the line, however, the width and contrast of the flame shadow may be used to infer qualitatively the absorptivity of sodium at this frequency and temperature. The sensitivity of the dye laser to manual wavelength adjustments is such that approximately three revolutions of a microdial linked to a diffraction grating controlling the dye laser frequency are required to span the absorption spectrum of one of the $\mathrm{D}$-lines. This resolution was found to be satisfactory for the purposes of the method and experiments presented here.

\section{4}

\section{Alternative sources of sodium seed}

Despite several disadvantages, sodium was used in the form of salt crystals grown on wires for most of this experimental study. Different ways to attach sodium chloride to the wire were tested, in the hope of developing a method that would minimize the possibility of sodium contamination of the flow outside the streaklines and create a source of atomic sodium that is not so brittle as to break off in the flow (a problem found in many of the runs with $\mathrm{NaCl}$ ).

Late in this development process, pure sodium instead of sodium chloride was placed at discrete positions on the wire. Despite the safety issues associated with sodium in its pure form (it reacts violently with water), there were advantages which made its use appealing for the purposes of flow tagging:

1. The paste-like nature of sodium allows small quantities to be directly attached to the wire, minimizing handling and reducing the risk of contamination.

2. Pure sodium eliminates the dissociation step previously required with $\mathrm{NaCl}$. Hence, higher concentrations of sodium may be expected to tag the flow. Also, this may extend the range of applicability of this technique by reducing the lower limit of temperature where the method works.

Pure sodium was used with success in the experiment, as shown in Fig. 5. This figure shows two streaks generated at a reservoir condition of $10 \mathrm{MJ} / \mathrm{kg}$. The streak closest to the body, barely visible in this picture, was made using the original $\mathrm{NaCl}$ crystal method. Above that streak, another streak was generated using sodium metal. This streak was placed in the region of high vorticity generation and exhibits some instability towards the end of the picture. Results such as these will be used in following sections to discuss the primary instability mode of the shear layer produced by curved shocks. 

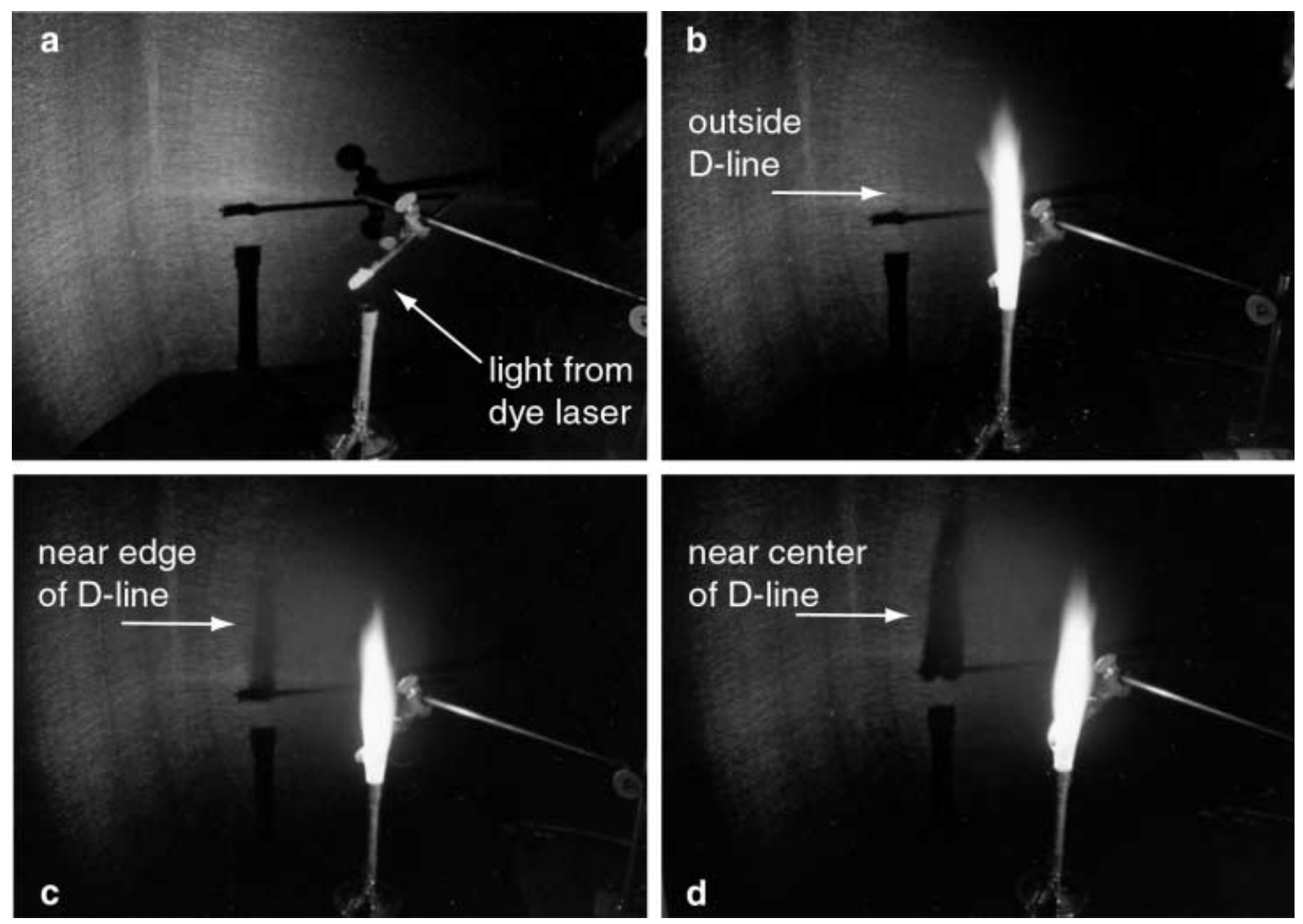

Fig. 4a d. Adjusting the dye laser frequency using a propane flame seeded with sodium. Image a shows the setup, with laser light

(coming from the right) illuminating a propane burner and casting its shadow on a screen. Above the burner is a metal rod with crystallized

Unfortunately, while it is now clear that sodium metal is better suited for the purposes of flow visualization than $\mathrm{NaCl}$, it was used for only a few shots towards the end of the study.

\section{5}

\section{Limits in the range of applicability of SWSV}

While there are no clear upper limits to the maximum enthalpy at which SWSV will work, the effectiveness of using sodium to visualize flows at low enthalpy is limited. For these flows, the width of the D-line is substantially reduced compared to higher enthalpy conditions, so that precise adjustment of the laser frequency becomes critical Moreover, the concentration of sodium in the flow is reduced at the lower temperatures, compounding the problem of detecting clear streaks in the flow. With our crude flame-tuning method, it has not been possible to observe streaklines at the lower enthalpy conditions $(5 \mathrm{MJ} / \mathrm{kg})$ in our experiments.

\section{4}

\section{The effect of the wire on SWSV results}

A drawback of SWSV is that it does not permit the investigation of the flow in a non-intrusive manner. In a study focused on flow stability (for which SWSV was originally developed), it is important to be able to separate possible wire effects from the stability of the shear layer itself.
$\mathrm{NaCl}$. In the subsequent shots, the dye laser is tuned: $\mathbf{b}$ several $\mathrm{m} \AA$ from the sodium $\mathrm{D}$ line; $\mathbf{c}$ near the edge of the $\mathrm{D}$ line, and $\mathbf{d}$ near the center of the D line. Note how absorption increases as the laser is tuned closer to the absorption peak

In the following sections, two effects of the wire on the flow are discussed: the interactions of the wire with the bow shock of the model and the effects of the wire wake on the flow.

\section{1}

\section{Interaction between wire and shock}

In order to seed specific areas of the flow and improve the reliability of the technique, it was found that placing the wire downstream of the bow shock of the model, directly into the shock layer, produced the best results. This procedure means that at some point along its length, the wire pierces the bow shock. Hence, the wire interacts with the shock in two ways: the shock generated by the wire interacts with the model shock and the wake behind the wire locally disturbs the model shock.

The combination of these two interactions was found to have an effect on some of the results obtained: in some cases, entrainment of sodium spanwise into the wire wake was observed. This was particularly evident when streaks were produced in the vicinity of the intersection of the wire with the shock and did not seem to depend significantly on the angle of attack of the wire. By investigating this region with SWSV, the critical region appeared to be localized to a few wire diameters around the point of intersection. At larger distances from the shock, the wire wake did not seem to have an effect on the flow. Within the critical region, however, streaks may be entrained into the wake of the wire, even across the shock, as shown in Fig. 6. 

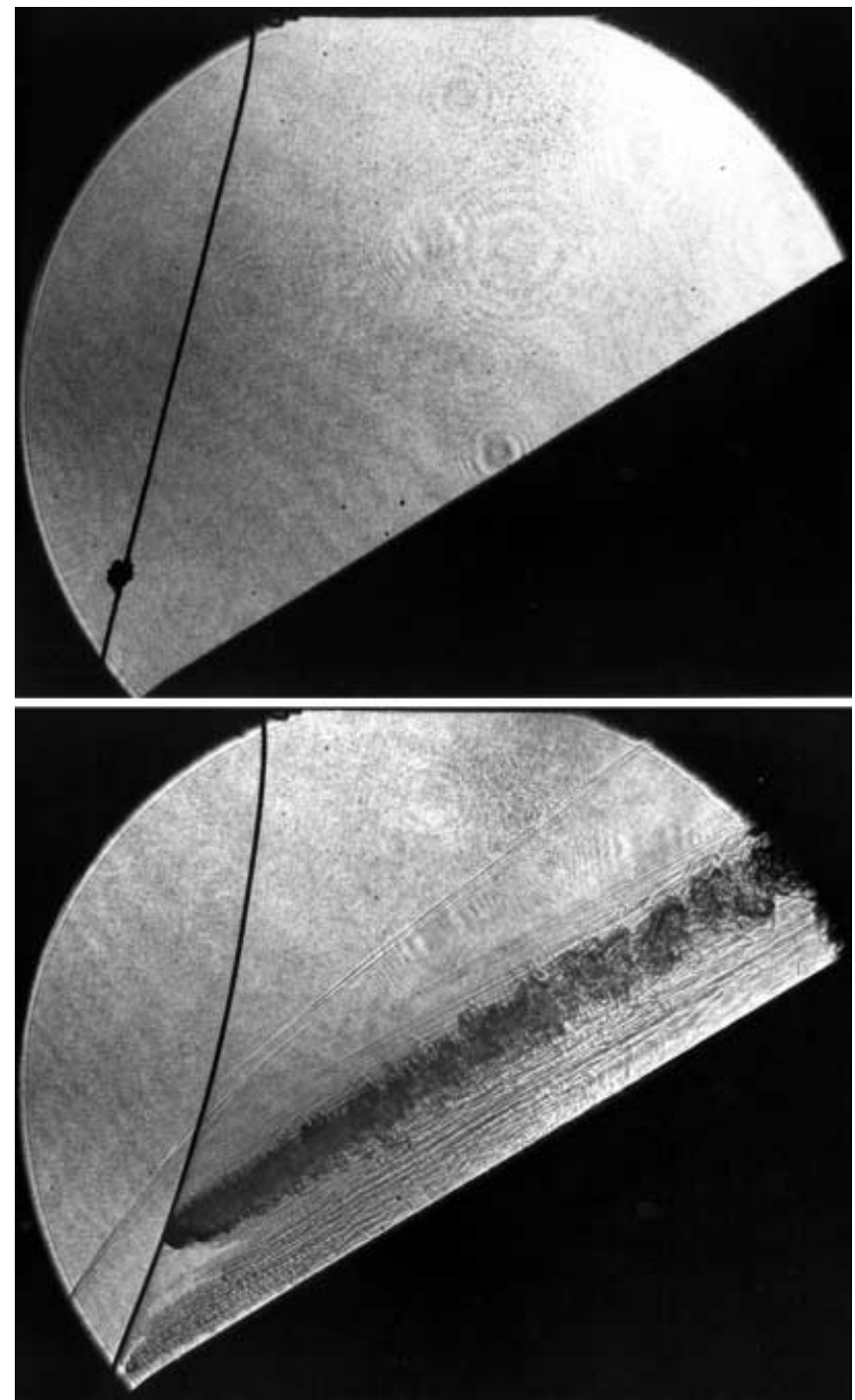

Fig. 5. Comparison of sodium lumps versus $\mathrm{NaCl}$ in tagging the flow. Top: wire setup before the shot. The sodium metal, pasted onto the wire, is clearly visible above the model. The salt crystal on the wire is to the left of the field of view. Bottom: shadowgraph of the flow over the model. The increased concentration of sodium along the streak generated by the metal lump is clearly evident when compared to the streak produced by the $\mathrm{NaCl}$ (below)

\section{2}

\section{Effect of wire wake on flow}

The effect of the wire on the flow is, of course, not limited to its intersection with the shock. Since the wire is essentially a cylinder in cross-flow, it may be responsible for any structures observed in a streakline. The geometry of the model and of the optical system being known, quantitative measurements can be made to verify whether any observed structures are, in fact, shed by the wire or may be attributed to a different source.

One of the clearest examples of the results of our investigation that may be used to discuss this situation is presented in Fig. 7. This shadowgraph shows the flow over the blunted wedge, with a leading edge of $3.5 \mathrm{~cm}$ radius (the wedge has a span of approximately $20 \mathrm{~cm}$ and a half-angle of $30^{\circ}$. The cylindrical leading edge is

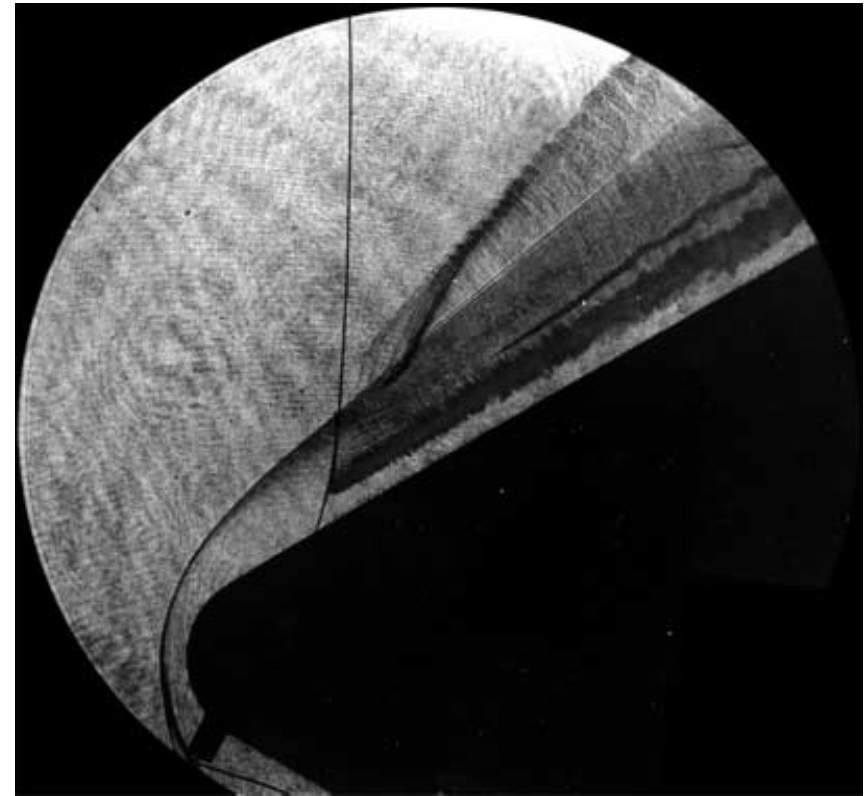

Fig. 6. Entrainment of a streakline within the wire wake, above the plane of the shock

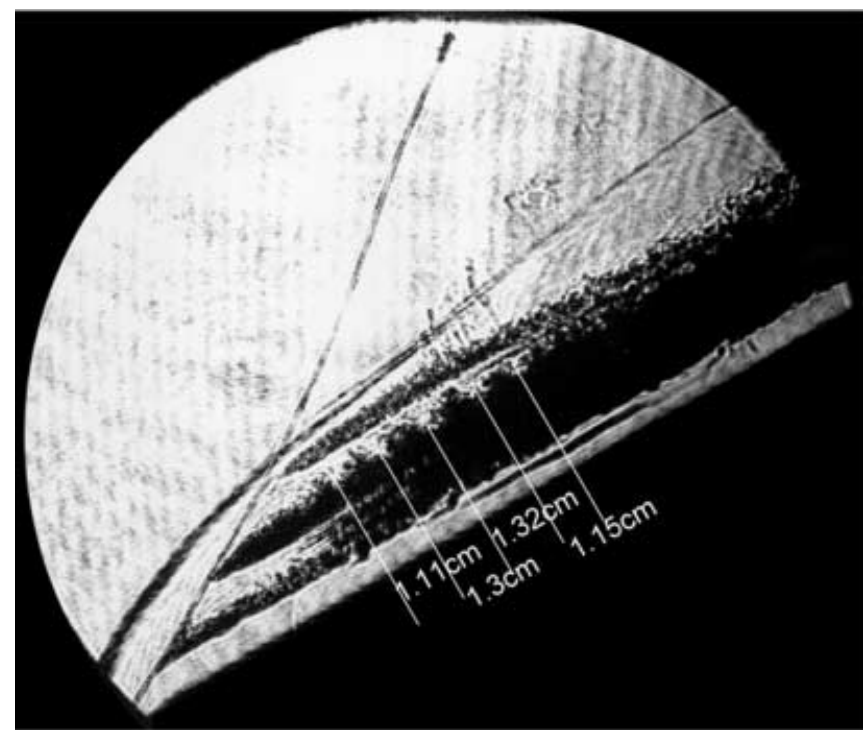

Fig. 7. Streaklines through the shear layer

removable.) The wire is $0.61 \mathrm{~mm}$ in diameter and has three sodium source points, spaced at $2.5-\mathrm{cm}$ intervals, spanning the shock layer from the body to the shock. The bottom streakline is aligned with a streamline near the stagnation point of the wedge; the center streakline is aligned approximately with the streamline that passes through the point of maximum vorticity generation (i.e., the center of the shear layer); and the top streakline is very close to the oblique (nearly straight) part of the shock.

\section{3}

\section{The cylinder in cross-flow}

The nominal free-stream conditions of Fig. 7 correspond to condition 1 in Table 1 . Using the actual T5 reservoir 
conditions recorded during the $\operatorname{shot}^{1}$. as input to a nonequilibrium nozzle flow program (see Lordi et al. 1966), better estimates of the flow conditions at the exit plane of the nozzle can be computed. This free-stream flow is then used as input into a computational fluid dynamics code for hypersonic flows in thermochemical non-equilibrium (Candler 1988), which computes the steady-state flow over the blunt wedge and provides estimates for the flow conditions downstream of the shock near the region where the wire seeds the flow. The code used here was selected based on a history of successful simulation of T5 flows, see e.g., Wen (1994) and Wen and Hornung (1995). Thus, the conditions corresponding to Fig. 7 are listed in Table 2.

The viscosity used for the wire Reynolds number calculation in Table 2 is computed using a formulation described by Blottner et al. (1971).

The cylinder in cross-flow is known to produce periodic oscillations in the wake of the cylinder over a large range of Reynolds numbers. The dimensionless frequency of these oscillations, the Strouhal number, is given by

$S=\frac{f d}{U_{\infty}}$,

where $f$ is the shedding frequency of the structures observed downstream of the cylinder and $d$ is the cylinder diameter. The value of the Strouhal number for flows over cylinders has been verified by many independent studies to remain approximately constant at $S=0.2$ for Reynolds numbers above $R e=200$ to as high as $R e=10^{7}$ (White 1991). Additional studies on flow over yawed cylinders (Van Atta 1968) have shown that this result is also applicable to cylinders inclined with respect to the flow if the Strouhal number is formed with the component of velocity normal to the cylinder.

Since the flow geometry and properties of Fig. 7 have been identified, a wire wake analysis may be performed to determine if the wavelength observed can indeed be due to the wire. The component of velocity normal to the wire is used to calculate the Strouhal number. Substituting the values pertaining to the middle streakline of Fig. 7 into Eq. 1, we get $S \approx 0.08$, well below the expected value for vortices shed from a cylinder. Furthermore, we observe that the two streaks emanating from above and below the center streak (corresponding to the center of the shear layer) do not exhibit the large structures present in the center streak. The variation in $U$ across the shear layer, responsible for variation in Reynolds number computed in Table 2, is not enough to vary that parameter outside the range for which the Strouhal number is constant. This suggests the hypothesis that the structures observed in the center streak are due to the instability of the shear layer.

Finally, we note that the component of velocity perpendicular to the wire in all of these streaks is only marginally subsonic for the center streak and supersonic for the top streak. It is important to consider, therefore, what

\footnotetext{
${ }^{1}$ Required specifically are the reservoir pressure and the reservoir temperature. The former is measured directly, while the latter may be computed from an equilibrium calculation across the reflected shock, performed here using a code written by McIntosh (1969)
}

Table 2. Local flow conditions at the source point of the three streaklines seen in Fig. 7

\begin{tabular}{lllllr}
\hline Streakline & $U(\mathrm{~m} / \mathrm{s})$ & $M$ & $\mathrm{~T}(\mathrm{~K})$ & $P(\mathrm{kPa})$ & $R e$ \\
\hline top & 2,850 & 2.4 & 4,400 & 108 & 1,200 \\
middle & 2,400 & 1.9 & 5,160 & 107 & 800 \\
bottom & 1,500 & 0.9 & 5,900 & 182 & 630 \\
\hline
\end{tabular}

happens to the stability of the flow in the wake of a cylinder at Mach numbers greater than 1 .

\section{The cylinder in supersonic flow}

Hot-wire studies in the wake of cylinders in supersonic flows have looked specifically at this problem (Behrens 1966). In these studies, the width of the outer wake behind the wire was measured and the spectrum of frequencies of instability was determined. The main instability mode, for given wire diameters, was found to occur in the far-wake of the cylinder in hypersonic flows (meaning in that region of the flow far from the coalescing free shear layer produced by the boundary layer separating around the cylinder and downstream from the wake shock). These instabilities developed over several hundred diameters downstream of the cylinder. It would appear therefore that the structures observed here cannot be due to an instability of the far-wake of the wire either, since they appear in the shadowgraph much closer to the wire.

\section{Wire vibration effect}

Another possible source for the vortices observed in Fig. 7 is a vibration of the wire itself, which might cause the vortex shedding to lock into this frequency. The natural frequency of such a wire is given by

$\omega_{n}=\frac{\pi \sqrt{ }(T / \delta)}{L}$

where $T$ is the tension in the wire, $\delta$ is the mass per unit length of the wire, and $L$ is the length of the wire.

For the case of Fig. $7, L \approx 28 \mathrm{~cm}$ and $\delta \approx 2.3 \mathrm{~g} / \mathrm{m}$. The tension in the wire, due to the drag of the flow and the lift of the prongs supporting it (they are at a $1^{\circ}$ angle of attack with respect to the flow in this figure), is estimated at approximately $20 \mathrm{~N}$. Substituting these values into Eq. 2,

$\omega_{n} \approx 1000 \frac{\mathrm{rad}}{\mathrm{s}}$,

which corresponds to a period of $6 \mathrm{~ms}$. This frequency is more than an order of magnitude lower than that required to explain the structures observed in Fig. 7 and therefore cannot be accountable for them. Structures caused by the wire vibrating in the flow would also be expected to affect all three streaklines, not just one as shown in Fig. 7 .

\section{4}

\section{An experimental control study}

In a further effort to investigate the effect of the wire on the structures observed, a control experiment was carried out. The goal of this control experiment was to isolate the role of the wake of the wire in flows similar to those of 


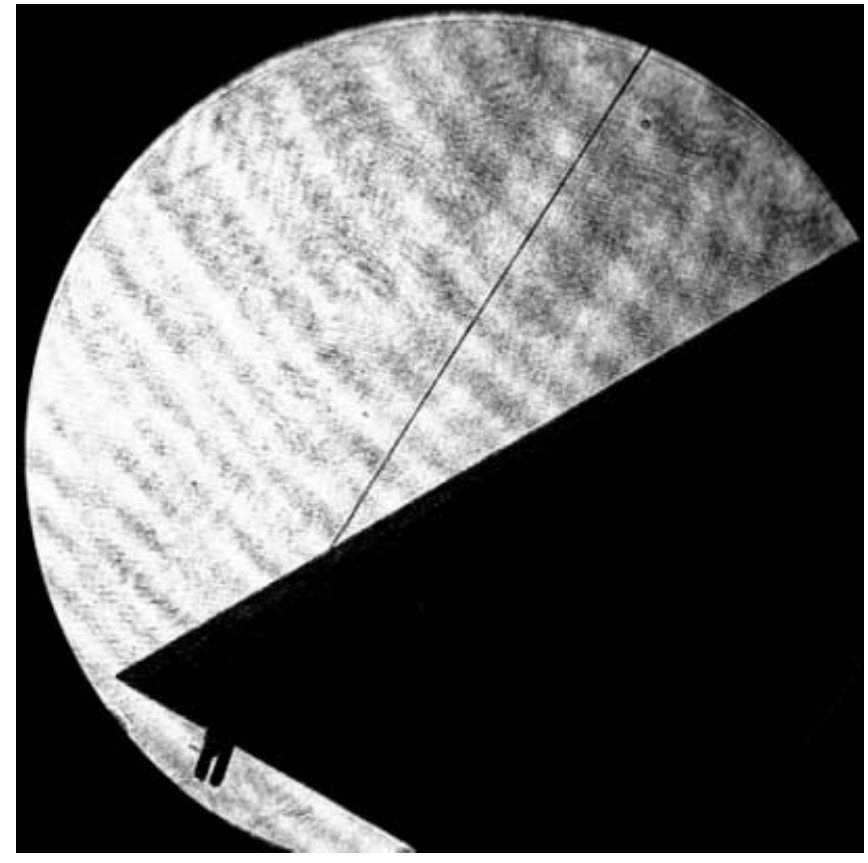

Fig. 8. Sharp leading edge setup for wire control study in T5. Note that the wire is fitted through the leading edge, so that the angle between the wire and the surface of the wedge is comparable to that of the experiment

interest here from that of the shock-produced shear layer by using a sharp wedge which produces a straight attached shock and therefore does not generate vorticity.

The test configuration of this experiment was similar to the one discussed so far and the flow condition was the same. The setup is shown in Fig. 8. With this configuration, shock curvature effects are eliminated from the flow and any streakline structure can only be caused by the effect of the wire. Wires of different diameters are used, so that flows over a range of wire Reynolds numbers can be compared.

The results of this experiment are shown in Fig. 9. They do not suggest that a significant broadening of the streak occurs as a result of the wire wake, nor do they exhibit a preferred scale of wake structures. As before (Fig. 7 top streak), the streaks exhibit turbulence in the wire wake. This is thought to be the turbulence usually observed in supersonic cylinder wakes close to the symmetry plane. The wavelength of structures that would be shed by a cylinder at a Strouhal number of 0.2 is included (to scale) in these figures. There does not appear to be a correlation with the wire diameter in any of the three shots in this figure, suggesting that no regular vortex shedding typical of incompressible flows over cylinders occurs, but that the flow is similar to the observations of Behrens (1966), who noted that instabilities did not develop until much further downstream in supersonic flow. This further supports our hypothesis that the structures observed in Fig. 7 are due to an instability in the shear layer that is produced by the curved shock.

Contrary to a free shear layer, which tends to grow rapidly in a direction normal to the flow, the shear layer produced by the curved shock is constrained on one side by the body surface and on the other by the shock, thus limiting its spatial growth. Nevertheless, by the end of the
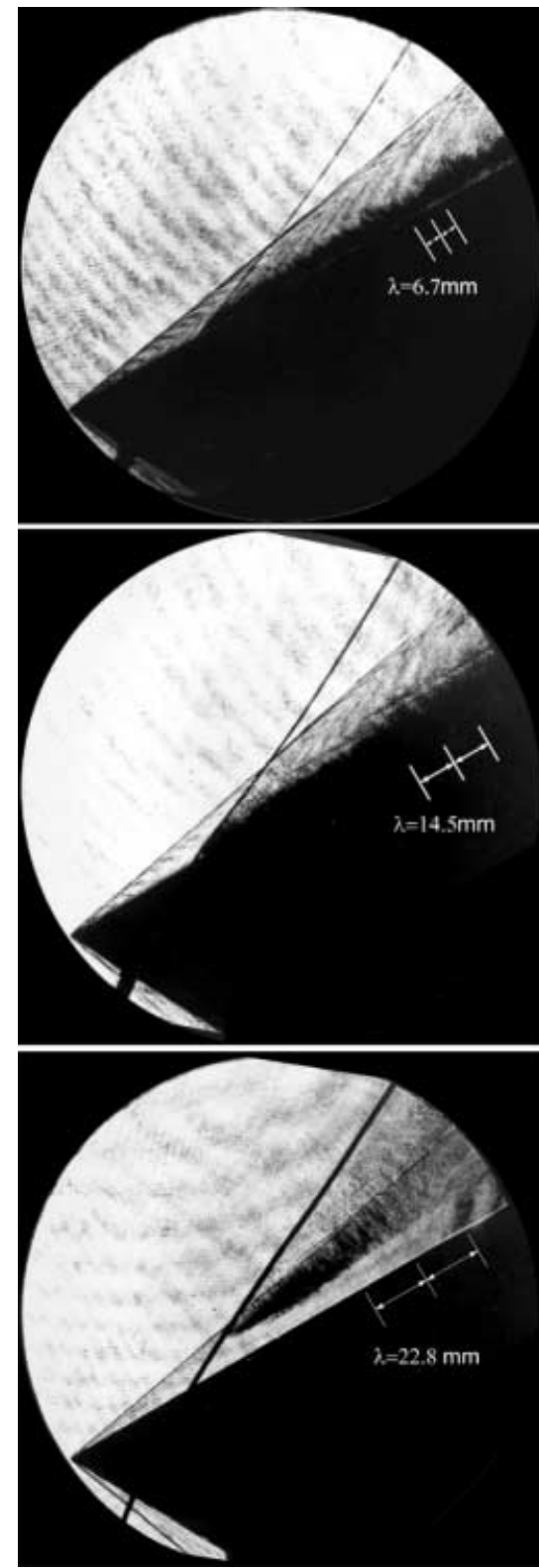

Fig. 9. Test of the wire wake instability. All three shots use condition 1 listed in Table 1 . This test examines the effect of wire diameter. It is $0.5 \mathrm{~mm}$ in the top picture; $1.0 \mathrm{~mm}$ in the middle and $2.0 \mathrm{~mm}$ in the bottom frame. The corresponding (expected) wavelength is shown to scale in the figures. Clearly, no structures of the expected scale occur

shadowgraph, the three streaks have completely mixed together. Also, the bottom streak (closest to the body), which is not in a region of high shear in the flow, remains relatively undisturbed for most of its trajectory.

\section{5}

Summary of results from SWSV

Streakline visualization was used in more than 50 runs with models of three different nose radii $(19,35$, and $63.5 \mathrm{~mm}$ ) at the three free-stream conditions of Table 1 . In approximately one-third of the cases, a preferred scale of structures could be measured clearly (category A). In another third, a preferred scale was evident in only two structures or had only small amplitude (category B). In the 


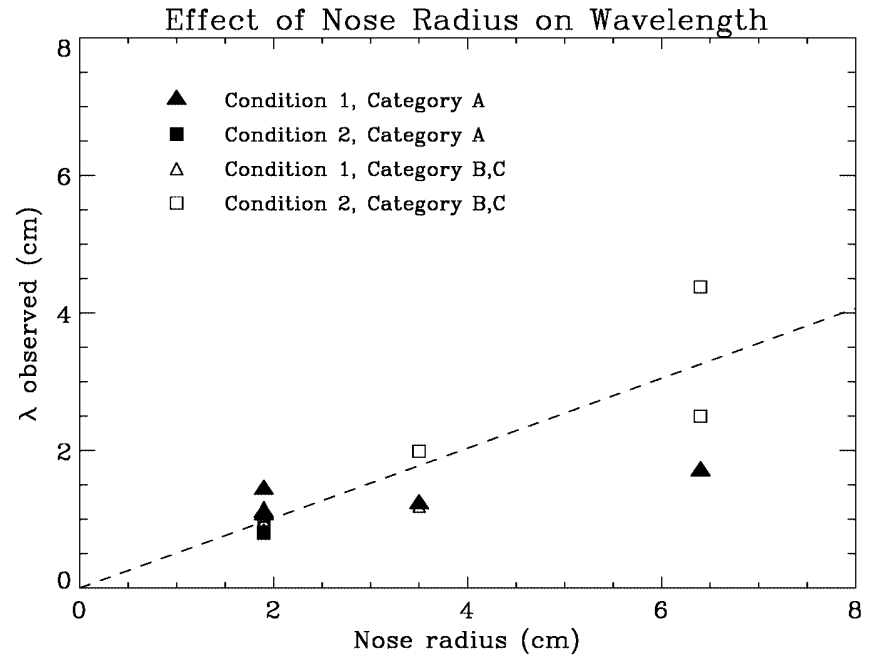

Fig. 10. Correlation between the wavelength of the structures observed in the shear layer and the nose radius

last third, only one or two structures could be discerned (category $\mathrm{C}$ ). In this application, the method does not produce particularly clean streaks and the central wake turbulence can obscure regular structures. If an instability of the shear layer produced by the curved shock does occur, the size of the structures has to scale with the nose radius, since, for the inviscid flow, this is the only characteristic length. To test this, the measured scale is plotted against the nose radius in Fig. 10.

The overall trend of these data shows an increase of wavelength with nose radius, but the scatter of the data that results from the error in measuring the wavelength, is too large to make conclusions about proportionality. Nevertheless, the dotted line of best fit suggests that the instability in the shear layer is most susceptible to disturbance wavelengths of about one-half of the leading edge radius.

While the results presented here are suggestive of an instability of the kind expected, they are not sufficiently convincing to draw firm conclusions. In part, this may be connected to the fact that the highest normal-shock density ratio achievable in $\mathrm{T} 5$ nozzle flows is approximately 12, whereas the work of Hornung and Lemieux (2001) has shown that the shear layer becomes unstable to small-amplitude disturbances within a few nose radii only when this density ratio is 14 or greater. The fact that we observe the instability in nozzle flows at all may be related to the disturbance amplitude in T5 nozzle flows. The wavelength of the instability observed by SWSV is in line with the results of Hornung and Lemieux (2001).

\section{6}

\section{Conclusion}

A technique for visualizing streaklines in hypervelocity flows, named SWSV, was developed for the purpose of investigating the stability of shear layers produced by curved shocks. The technique uses the absorption and enhanced refractivity properties of sodium near one of the D-lines to visualize the streaklines. The effect of the wire on the flow was investigated and found to be unimportant to the purpose of the investigation.

SWSV was used in a series of experiments using hemicylindrically blunted wedges and showed that the shear layer produced by curved shocks may become unstable at sufficiently high density ratios and disturbance level. The preferred wavelength of the structures that develop in the shear layer is comparable to one-half of the leading edge radius of the wedge. The results obtained were corroborated in a numerical study published separately.

\section{References}

Behrens FW (1966) Flow field and stability of the far wake behind cylinders at hypersonic speeds. PhD thesis. California Institute of Technology, Pasadena, Calif.

Bélanger J (1993) Studies of mixing and combustion in hypervelocity flows with hot hydrogen injection. PhD thesis. Graduate Aeronautical Laboratories, California Institute of Technology, Pasadena, Calif.

Blendstrup G, Bershader D, Langhoff P (1979) Recent results of resonant refractivity studies for improved flow visualization. In: Lifshitz A, Rom J (eds) Proceedings of the 12th International Symposium on Shock Waves, 1979, Jerusalem, Israel, pp 258265

Blottner FG, Johnson M, Ellis M (1971) Chemically reacting viscous flow program for multi component gas mixtures. Technical Report SC RR 70 754. Sandia Laboratories, Albuquerque, N. M.

Candler G (1988) The computation of weakly ionized hypersonic flows in thermo chemical nonequilibrium. PhD thesis. Stanford University, Stanford, Calif.

Cummings EB (1995) Laser induced thermal acoustics. PhD thesis. Graduate Aeronautical Laboratories, California Institute of Tech nology, Pasadena, Calif.

Fric TF, Roshko A (1994) Vortical structures in the wake of a trans verse jet. J Fluid Mech 279:1

Germain P (1994) The boundary layer on a sharp cone in high enthalpy flow. PhD thesis. Graduate Aeronautical Laboratories, California Institute of Technology, Pasadena, Calif.

Goddard VP, MacLaughlin JA, Brown FNM (1959) Visual supersonic flow patterns by means of smoke lines. J Aero/Space Sci 26:761 762

Hayes WD, Probstein RF (1966) Hypersonic flow theory, 2nd edn. Academic Press, New York

Hornung HG, Lemieux P (2001) Shock layer instability near the Newtonian limit of hypervelocity flows. Phys Fluids 13:2394

Lordi JA, Mates RE, Moselle JR (1966) Computer program for the numerical solution of nonequilibrium expansions of reacting gas mixtures. Technical Report NASA CR 472. NASA, Washington, D.C.

Lukasiewicz J (1973) Experimental methods of hypersonics. Marcel Dekker, New York

McIntosh MK (1969) A computer program for the numerical calcu lation of equilibrium and perfect gas conditions in shock tunnels. Technical Note CPD 169. Australian Defense Scientific Service, Canberra, Australia

Thorne AP (1988) Spectrophysics. Chapman \& Hall, London

Atta CW Van (1968) Experiments on vortex shedding from yawed circular cylinders. AIAA J 6:931

Wen CY (1994) Hypervelocity flow over spheres. PhD thesis. Graduate Aeronautical Laboratories, California Institute of Technology, Pasadena, Calif.

Wen CY, Hornung HG (1995) Non equilibrium dissociating flow over spheres. J Fluid Mech 299:389

White FM (1991) Viscous fluid flow, 2nd edition. McGraw Hill, New York 